\title{
Frecuencia de síndrome metabólico en pacientes con diagnóstico previo de diabetes gestacional
}

\author{
Terrones-Saldívar Ma del Carmen*, Ramírez-Salado Diana Paola**, Prieto-Macías Jorge*, Reyes- \\ Robles Martha Elena***, Rosas-Cabral Alejandro*
}

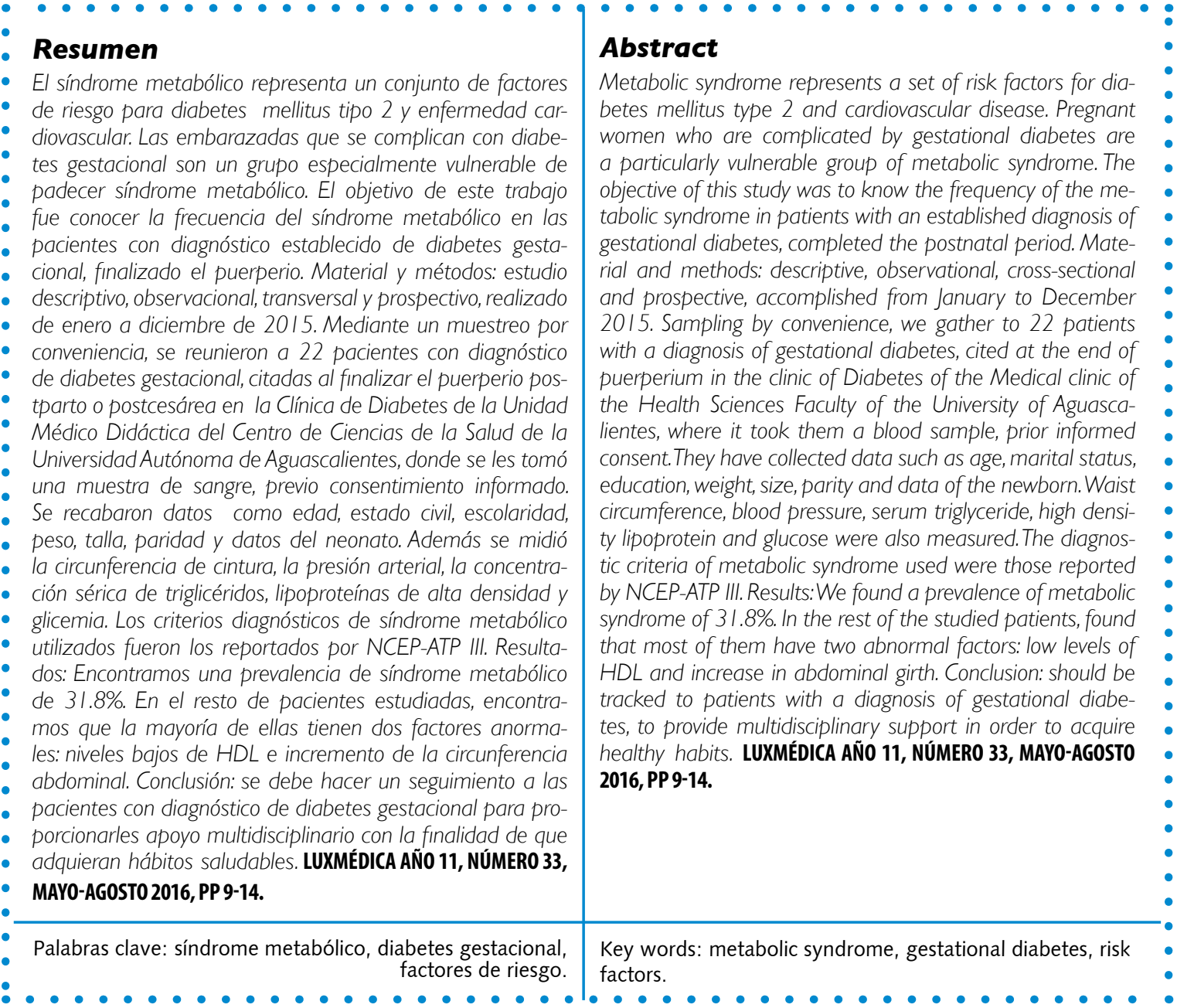

* Profesores investigadores del Centro de Ciencias de la Salud de la Universidad Autónoma de Aguascalientes.

** Estudiante del décimo semestre de la carrera de Medicina del Centro de Ciencias de la Salud de la Universidad Autónoma de Aguascalientes.

*** Técnico de apoyo

Fecha de recibido: 10 de diciembre 2015

Fecha de aceptación: 28 de febrero 2016

Correspondencia: Dra en C Ma del Carmen Terrones Saldívar. Edificio 107, planta alta, Centro de Ciencias de la Salud, Universidad Autónoma de Aguascalientes. Avenida Universidad \#940, código postal 20131. Aguascalientes, Ags., México. Teléfono (449) 9108443. Correo electrónico mcterron@correo.uaa.mx 


\section{Introducción}

Se denomina síndrome metabólico al conjunto de alteraciones metabólicas constituido por obesidad de distribución central, disminución de las concentraciones del colesterol unido a las lipoproteínas de alta densidad ( $\mathrm{cHDL}$ ), elevación de las concentraciones de triglicéridos, aumento de la presión arterial (PA) e hiperglucemia. ${ }^{1}$ El SM es un tema de gran importancia debido a que es un antecedente de las dos primeras causas de muerte en la población mexicana (enfermedades cardiovasculares y diabetes mellitus tipo 2). ${ }^{2,3}$

La diabetes gestacional (DG) se define como la intolerancia a los carbohidratos que conduce a la hiperglucemia con inicio o primer reconocimiento durante el embarazo. ${ }^{4}$ Hasta en un $90 \%$ de las mujeres con DG la intolerancia a los carbohidratos desaparecerá después del embarazo, sin embargo, diversos estudios demuestran que hasta un tercio de las mujeres afectadas progresarán en un futuro a diabetes o alteraciones del metabolismo de la glucosa, y en un lapso de 5 a 16 años del parto desde un 17\% hasta un $63 \%$ habrán desarrollado alteraciones del metabolismo. ${ }^{5,6}$ Las mujeres con DG tienen un riesgo muy alto de desarrollar diabetes en etapas posteriores de la vida y un riesgo de SM 3 veces mayor. ${ }^{7,8}$ La DG tiene una prevalencia aproximada del 2-16\%.7 Actualmente existe la hipótesis de que la diabetes gestacional es una manifestación temprana del SM en la mujer. ${ }^{9}$ En el embarazo existe una resistencia a la insulina (RI) y una hiperinsulinemia que puede predisponer a algunas mujeres a desarrollar diabetes durante la gestación. La hiperinsulinemia es la anomalía más precoz observada en la diabetes mellitus tipo 2 , produciéndose posteriormente un incremento de la producción hepática de glucosa, conduciendo a la hiperglucemia. Dicha insulinorresistencia se favorece por la secreción placentaria de hormonas diabetógenas (hormona del crecimiento, cortisol, lactógeno placentario, progesterona), así como por el aumento de la adiposidad materna. ${ }^{10}$ Hoy en día, se sabe ampliamente que el síndrome metabólico es un factor de riesgo múltiple para enfermedades cardiovasculares y metabólicas. ${ }^{8}$ El objetivo general fue conocer la frecuencia del síndrome metabólico postparto en pacientes con diagnóstico previo de diabetes gestacional.

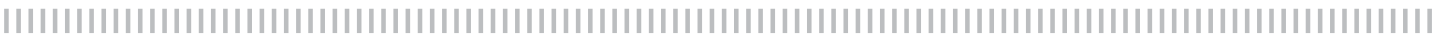

\section{Material y métodos}

Se realizó un estudio descriptivo, prospectivo y transversal, de enero a diciembre 2015. Se incluyeron 22 pacientes, se tomó como criterio de inclusión que fueran pacientes con diagnóstico establecido de diabetes gestacional de acuerdo a los criterios de la Asociación Americana de Diabetes (curva de tolerancia oral a la glucosa de 3 horas y 100 gr de glucosa con dos o más valores alterados, entre la semana
24 y 28 del embarazo) y la resolución del embarazo realizada en hospital de segundo nivel de atención. A partir del inicio de la inclusión de pacientes, se llevó a cabo la siguiente rutina: antes de su egreso se llenó la hoja de recolección de datos y se les dio una cita a las ocho semanas de postparto a la Clínica de Diabetes de la Unidad Médico Didáctica de la Universidad Autónoma de Aguascalientes, donde se les tomó una muestra de sangre para la cuantificación 
de colesterol, triglicéridos, HDL y glicemia. Con los resultados de laboratorio y la revisión a la paciente, se pudo identificar a las pacientes que reunieron características de diagnóstico de síndrome metabólico. En el día de la cita en consulta externa, se les midió la circunferencia abdominal y se midieron las cifras de presión arterial. Se recabaron datos de su expediente clínico. Se obtuvo el consentimiento informado a todas las pacientes que quedaron incluidas en este trabajo de investigación. Los criterios diagnósticos utilizados para SM fueron los publicados por el National Cholesterol Education Program (NCEP) Adult Treatment Panel-III (NCEP-ATP III). Las pacientes que tuvieron 3 de los 5 criterios siguientes fueron diagnosticadas con Síndrome Metabólico:

1. Circunferencia abdominal mayor o igual a $88 \mathrm{~cm}$.

2. Elevación de los triglicéridos mayor o igual a $150 \mathrm{mg} / \mathrm{dl}$

3. Disminución de la HDL colesterol, menor a $50 \mathrm{mg} / \mathrm{dl}$

4. Elevación de la presión arterial mayor o igual a $130 \mathrm{mmHg}$ la sistólica y mayor o igual a $80 \mathrm{mmHg}$ la diastólica.

5. Elevación de la glicemia sanguínea en ayunas mayor o igual a $100 \mathrm{mg} / \mathrm{dl}$.

Los resultados obtenidos se expresan en medidas de tendencia central, porcentajes y promedios.

\section{| | | | | | | | | | | | | | | | | | | | | | | | | | | | | | | | | | | | | | | | | | | | | | | | | | | | | | | | | | | | | | | | | | | | | | | | | | | | | | | | | | | | | | | | | | | | | | | | | | | | | | | | | | | | | |}

\section{Resultados}

El promedio de edad de las 22 pacientes estudiadas fue de $23.2 \pm 3.1$ años (rango de 18 a 30 años). El antecedente familiar de diabetes por línea materna o paterna lo encontramos positivo en la mayoría de las pacientes $(81.8 \%)$. El estado civil que predominó fue la unión libre $(72 \%)$. En general, encontramos un nivel básico de escolaridad, con la preparatoria completa en el $45 \%$ de las pacientes; no hubo analfabetas ni tampoco pacientes con carrera universitaria. El nivel socioeconómico bajo fue lo manifestado por todas las pacientes. El pro- medio del peso previo al embarazo fue de $68.1 \mathrm{~kg}(55-109 \mathrm{Kg})$, mientras que el peso actual registrado fue de $76.1 \pm 12.3 \mathrm{Kg}(60-$ $102.5 \mathrm{~kg})$. La talla promedio encontrada en las pacientes fue de $1.65 \pm 0.05 \mathrm{~m}$ (1.50 a $1.75 \mathrm{~m}$ ) y el índice de masa corporal (IMC) promedio fue de $27.9 \pm 3.4$ (rango 20-34), este resultado corresponde a sobrepeso. Desglosando estos resultados, encontramos que el $36.3 \%$ tuvieron el IMC menor de 25 , es decir, en su peso normal, mientras que el $27.2 \%$ fueron identificadas con sobrepeso (IMC entre 25 y 29.9), y el $36.3 \%$ con obesidad (IMC entre 30 y 39.9) (figura 1).

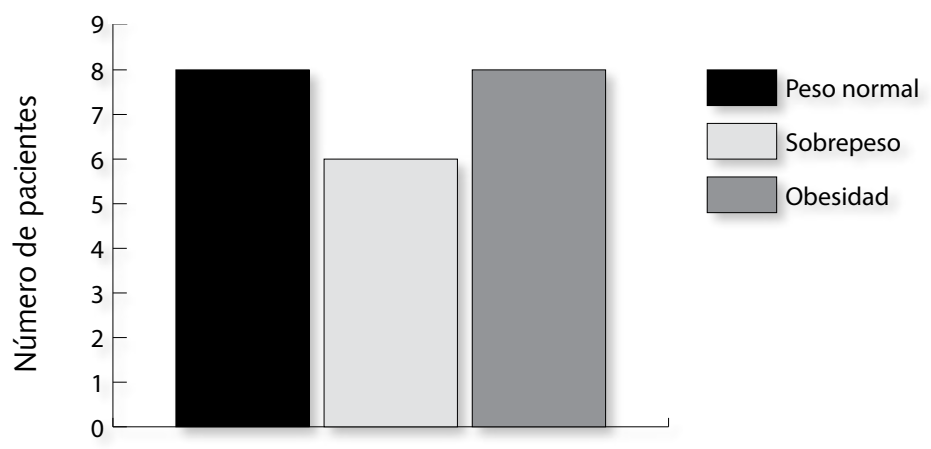

Figura 1. Distribución de las pacientes estudiadas al finalizar el puerperio con diagnóstico previo de diabetes gestacional, deacuerdo a su Índice de Masa Corporal. 
En cuanto a la paridad de las pacientes, encontramos en orden decreciente, la secundigesta $(54.5 \%)$, multigesta $(27.2 \%)$ y primigesta (18.1\%). Entre las complicaciones perinatales previas, encontramos como antecedente más frecuente la diabetes gestacional en casi una de cada tres pacientes estudiadas (31.5\%). Predominó la interrupción del embarazo por vía abdominal en la mayoría de los casos (72.7\%) La indicación de las cesáreas, aunado a su diagnóstico de diabetes gestacional, fue: placenta previa, macrosomía, trabajo de parto prolongado e inducto-conducción fallida. Entre los neonatos predominó el sexo masculino en el $68.1 \%$ de los casos. La talla y el peso promedio fue de $51.5 \pm$ $2.8 \mathrm{~cm}$ y $3350 \pm 380 \mathrm{gr}$, respectivamente. Hubo un neonato con sepsis y uno con dificultad respiratoria, que evolucionaron satisfactoriamente y fueron dados de alta por mejoría.

En la muestra estudiada la prevalencia del síndrome metabólico fue de $31.8 \%$. En la mayoría de las pacientes (90.9\%), encontramos bajos los niveles de HDL (menores a $50 \mathrm{mg} / \mathrm{dL}$ ), representando el factor de riesgo más frecuente (promedio de $39.3 \mathrm{mg} /$ dl). En segundo lugar, la medición de la circunferencia de la cintura mayor a $88 \mathrm{~cm}$ la encontramos en el $72.7 \%$ de las pacientes (promedio de $97.8 \mathrm{~cm}$ ). La glicemia en ayuno superior a $100 \mathrm{mg} / \mathrm{dl}$, la identificamos en cinco pacientes (22.7\%) (figura 2 ). En todos los casos se registraron cifras tensionales normales. Es de llamar la atención que sólo dos paciente no tuvieron ningún factor de riesgo para el síndrome metabólico. En más de la mitad de las pacientes (54.5\%) se identificaron dos factores.

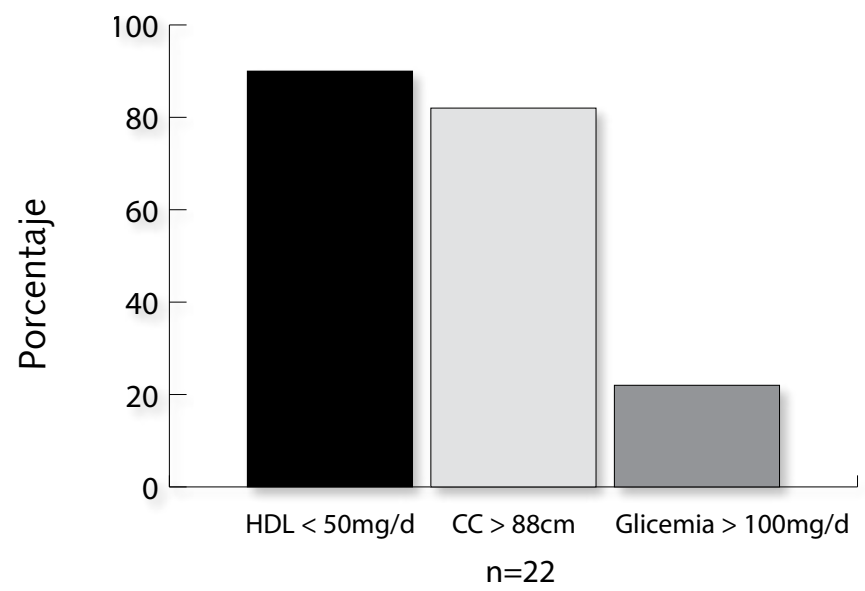

Figura 2. Distribución de los tres factores más frecuentes que integraron el diagnóstico de síndrome metabólico en pacientes con diabetes gestacional previa.

\section{| | | | | | | | | | | | | | | | | | | | | | | | | | | | | | | | | | | | | | | | | | | | | | | | | | | | | | | | | | | | | | | | | | | | | | | | | | | | | | | | | | | | | | | | | | | | | | | | | | |}

\section{Discusión}

Nuestros resultados arrojan información que debe ser tomada con ciertas consideraciones, ya que una limitación de este estudio fue la muestra reducida. Encontramos que la frecuencia del síndrome metabólico en la muestra estudiada fue de $31.8 \%$, y no es sorprendente que el sobrepeso-obesidad (reflejada a través de la medición de la circunferencia de la cintura), sea un dato anor- mal identificado en la mayoría de las pacientes, dado que actualmente es considerado como uno de los principales problemas de salud pública de nuestro país. ${ }^{13}$ Comparando la prevalencia de nuestra muestra, observamos que es menor a la reportada por otros autores, como el grupo de Ferrada y col, de Chile ${ }^{14}$, que publicaron en el 2007 una prevalencia del $55.1 \%$ en un grupo de 58 pacientes, mientras que el grupo de 
Wender y colaboradores en Polonia ${ }^{15}$ en el mismo año, encontraron un frecuencia de $58.3 \%$. Otros autores han reportado que el IMC, el sobrepeso/obesidad y el antecedente familiar de diabetes mellitus son factores que incrementan el riesgo de síndrome metabólico. ${ }^{16}$

Los tres factores de riesgo más frecuentes encontrados en nuestro estudio, fueron HDL menor de $50 \mathrm{mg} / \mathrm{dl}$, circunferencia de la cintura mayor de $88 \mathrm{~cm}$ y glicemia mayor de $100 \mathrm{mg} / \mathrm{dl}$, en orden de mayor a menor, respectivamente (figura 2 ). Comparando estos resultados con lo reportado por Edalat ${ }^{17}$ y colaboradores (2013) observamos que ellos encontraron a la obesidad abdominal como el factor más frecuente encontrado entre sus pacientes con DG previa (60\%), seguido de la disminución de HDL (en el $51 \%$ ) y la hipetensión (29\%). Nos llama la atención que el porcentaje de HDL y obesidad abdominal es mayor en este estudio que en el Edelat $(90.9 \%$ vs $51 \%$ y $60 \%$ vs $81.8 \%$ respectivamente). En otro estudio realizado en Venezuela siguieron a las pacientes con DG previa durante 3,5 \pm 2,6 años, encontraron que cerca de la mitad de las mujeres estudiadas reunían los criterios ATPIII para SM, donde destacaron a la obesidad como el mejor predictor del mismo. ${ }^{18}$ En los últimos años se han publicado estudios que han observado un incremento en el riesgo de síndrome metabólico en pacientes con historia de diabetes gestacional. ${ }^{19,20}$ En un meta-análisis realizado por Xu ${ }^{21}$ y colaboradores (2014) que incluyó 17 artículos concluyeron que las mujeres con DG previa tiene un riesgo casi cuatro veces mayor de síndrome metabólico, que se incrementa aún más cuando tienen un IMC elevado (OR 5.39; IC 95\%, 4.47 a 6.50).

Como podemos observar algunos componentes metabólicos asociados al síndrome metabólico son causantes de la diabetes gestacional, de tal manera que algunos autores han propuesto considerar a la DG como un componente del SM: esta perspectiva ofrecería un modelo de estudio temprano para la prevención de complicaciones metabólicas y cardiovasculares futuras, como lo proponen diferentes investigadores. ${ }^{14}, 22-25$ El reconocimiento de este síndrome durante el embarazo podría ayudar a identificar a un subgrupo de mujeres quienes no solamente pueden desarrollar complicaciones durante el embarazo, sino que, potencialmente, tienen un riesgo incrementado de condiciones metabólicas y cardiovasculares a lo largo de su vida. ${ }^{9}$

\section{Conclusiones}

Siendo el sobrepeso y la obesidad uno de los dos factores de riesgo más frecuente en las pacientes con síndrome metabólico en la muestra de mujeres con diabetes gestacional previa, y considerando que es susceptible de modificación, entonces resalta la importancia de identificar y dar seguimiento a las pacientes con diabetes gestacional para que inicien la modificación de los estilos de vida a través de intervenciones multidisciplinarias, con apoyo médico, nutricional, de activación física y psicológico para evitar que tarde o temprano, padezcan alguna de las enfermedades crónico-degenerativas que ocasionan las principales causas de morbimortalidad en nuestro país.

Agradecimiento

Se agradece al personal de laboratorio de la Unidad Médica Didáctica por su apoyo. 


\section{Bibliografía}

1. Zimmet, P. Alberti, G. \& Serrano, M. (2005) Una nueva definición mundial del síndrome metabólico propuesta por la Federación Internacional de Diabetes: fundamento y resultados. Rev Esp Cardiol. 2005;5858(12):1371-6. DOI: 10.1016/S03008932(05)74065-3.

2. García-García C. Diabetes mellitus gestacional. Med Int Mex 2008; 24(2):148-56.

3. Instituto Nacional de Estadística y Geografía (México). Mujeres y hombres en México 2013/Instituto Nacional de Estadística y Geografía.México: INEGI, c201 disponible http://www.inegi.org.mx/prod serv/contenidos/espanol/bvinegi/productos/integracion/sociodemografico/mujeresyhombres/2013/ Myh 2013.pdf.

4. Göbl S. Chistian, Latife B., Prikoszovich T., Winzer, C., Pacini, G., Willer, K., Early Possible Risk Factor for Overt Diabetes After Gestacional Diabetes Mellitus. Obstetrics \& Gynecology. 2011;11(1):71-78 doi: 10.1097/AOG.0b013e318220e18f.

5. Hunger-Date W, Mosebach N, Sámann A, et al. Prevalence of impaired glucose tolerance 6 years after gestational diabetes. Exp Clin Endocrinol Diabetes 2006; 114:11-17.

6. Hopmans TE, van Houten $C$, Kasius A, Kouznetsova OI, Nguyen LA, Rooijmans SV, Voormolen DN, van Vliet EO, Franx A, Koster MP. Increased risk of type II diabetes mellitus and cardiovascular disease after gestational diabetes mellitus: a systematic review Ned Tijdschr Geneeskd. 2015;159:A8043.

7. England LJ, Dietz PM, Njoroge T, et al. Preventing type 2 diabetes: public health implications for women with a history of gestational diabetes mellitus. Am J Obstet Gynecol 2009;200:365.e1-365.e8.

8. Archambault, C., Arel, R., \& Filion, K. B. (2014). Gestational diabetes and risk of cardiovascular disease: a scoping review. Open Medicine, 8(1), e1-e9.

9. Yépez MC, Zeppenfel ME, Colón JA, Zimmer E. Síndrome metabólico durante el embarazo. Complicaciones materno-fetales. Rev Obstet Ginecol Venez 2011; 71(2):77-87.

10. Gallo JL, Díaz-López MA, Gómez-Fernández J, Hurtado F, Presa JC y Valverde M. Síndrome metabólico en obstetricia. Clin Invest Gin Obst 2010; 37 (6): 239-246.

11. Bartha JL, González-Bugatto F, Fernández-Macías $\mathrm{R}$, González-González NL, Comino-Delgado $\mathrm{R}$, Hervías-Vivancos B. Metabolic syndrome in normal and complicated pregnancies. European Journal of Obstetrics \& Gynecology and Reproductive Biology 2008; 137:178-184.

12. Eckel RH, Cornier M-A. Update on the NCEP ATP-III emerging cardiometabolic risk factors. BMC Medicine. 2014;12:115. doi:10.1186/1741-7015-12-115.

13. Córdova-Villalobos J A, Sobrepeso y obesidad, problemas de salud pública en México. Cirugía y Cirujanos 200977421-422. Disponible en: http://www. redalyc.org/articulo.oa?id $=66212714001$. Fecha de consulta: 11 de diciembre de 2015.

14. Ferrada C, Molina M, Cid L, Riedel G, Ferrada C, Arévalo R. Relación entre diabetes gestacional y síndrome metabólico. Rev Med Chile 2007; 135: 15391545.

15. Wender-Ozegowska E1, Sporna $M$, Zawiejska A, Sporna A, Brazert J. Components of metabolic syndrome in women after gestational diabetes. Pol Arch Med Wewn. 2007 Oct;117(10):457-62.

16. Masuzaki $\mathrm{H}$, Paterson J. A transgenic model of visceral obesity and the metabolic syndrome. Science 2001;294:2166-2170.

17. Edalat B, Sharifi F, Badamchizadeh Z, HosseinNezhad A, Larijani B, Mirarefin M, Fakhrzadeh $\mathrm{H}$. Association of metabolic syndrome with inflammatory mediators in women with previous gestational diabetes mellitus. J Diabetes Metab Disord. 2013 Jan 22;12(1):8. doi: 10.1186/2251-6581-12-8.

18. Rivas Aleida M, González Julio C, Guevara Mary C, Dávila Solange G. Alteraciones clínico-metabólicas en mujeres con diabetes gestacional previa. Rev Obstet Ginecol Venez [Internet]. 2010 Mar [citado 2015 Dic 11] ; 70( 1 ): 18-23. Disponible en: http://www.scielo.org.ve/scielo.php?script =sci arttext\&pid=S0048-77322010000100004\&Ing=es.

19. G. Di Cianni, C. Lencioni, L. Volpe, A. Ghio, I. Cuccuru, G. Pellegrini, L. Benzi, R. Miccoli, S. Del Prato. Creactive protein and metabolic syndrome in women with previous gestational diabetes .2007;23(2):135140 DOI: 10.1002/dmrr.661.

20. Tam WH, Ma RC, Yang X, Ko GT, Lao TT, Chan MH, Lam CW, Cockram CS, Chan JC. Cardiometabolic risk in Chinese women with prior gestational diabetes: a 15-year follow-up study. Gynecol Obstet Invest. 2012;73(2):168-76. doi: 10.1159/000329339.

21. Xu, Y., Shen, S., Sun, L., Yang, H., Jin, B., \& Cao, X. (2014). Metabolic Syndrome Risk after Gestational Diabetes: A Systematic Review and Meta-Analysis. PLoS ONE, 9(1), e87863. http://doi.org/10.1371/ journal.pone.0087863.

22. Anila Verma, Charlotte M. Boney, Richard Tucker, and Betty R. Vohr. Insulin Resistance Syndrome in Women with Prior History of Gestational Diabetes Mellitus The Journal of Clinical Endocrinology \& Metabolism 2002 87:7, 3227-3235.

23. Akinci B, Celtik A, Yener S, Yesil S. Prediction of developing metabolic syndrome after gestational diabetes mellitus. Fertil Steril. 2010 Mar 1;93(4):124854. doi: 10.1016/j.fertnstert.2008.12.007.

24. Romero-Gutierrez G, Macías-Rocha AL, Puente-Álvarez El. Prevalencia de alteraciones en la tolerancia a la glucosa postparto en pacientes con diabetes gestacional previa. Ginecol Obstet Mex 2012; 80(10):631-636.

25. Nava $P$, Garduño $A$, Pestaña $S$, Santamaría $M$, Vázquez G, Camacho R, Herrera J. Obesidad pregestacional y riesgo de intolerancia a la glucosa en el embarazgo y diabetes gestacional. Rev Chil Obstet Ginecol 2011; 76(1):10-14 